\title{
Combining Graphics Processing Units, Simplified Time-Dependent Density Functional Theory, and Finite-Difference Couplings to Accelerate Nonadiabatic Molecular Dynamics
}

\author{
Laurens D. M. Peters, Jörg Kussmann, and Christian Ochsenfeld*
}

Cite This: J. Phys. Chem. Lett. 2020, 11, 3955-3961

Read Online

ACCESS | 㭊 Metrics \& More 回 Article Recommendations

ABSTRACT: Starting from our recently published implementation of nonadiabatic molecular dynamics (NAMD) on graphics processing units (GPUs), we explore further approaches to accelerate $a b$ initio NAMD calculations at the time-dependent density functional theory (TDDFT) level of theory. We employ (1) the simplified TDDFT schemes of Grimme et al. and (2) the Hammes-Schiffer-Tully approach to obtain nonadiabatic couplings from finite-difference calculations. The resulting scheme delivers an accurate physical picture while virtually eliminating the two computationally most demanding steps of the algorithm. Combined with our GPU-based integral routines for SCF, TDDFT, and TDDFT derivative calculations, NAMD simulations of systems of a few hundreds of atoms at a reasonable time scale become accessible on a single compute node. To demonstrate this and to present a first, illustrative example, we perform TDDFT/MM-NAMD simulations of the rhodopsin protein.

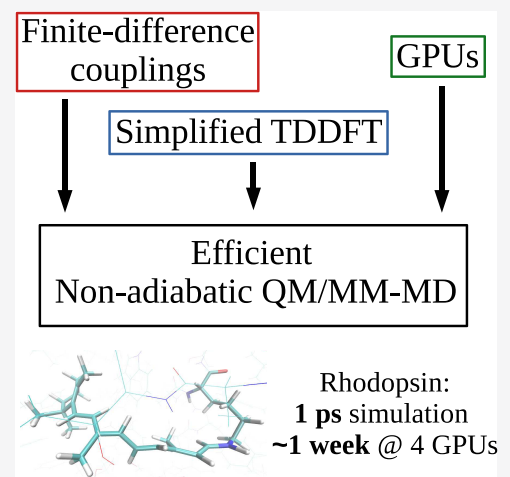

$\mathrm{N}$ onadiabatic molecular dynamics (NAMD) simulations using trajectory surface hopping (TSH $)^{1-4}$ have become a powerful tool to describe the dynamics of molecular systems involving multiple electronic states. Their field of application ranges from the description of rather small molecular machines $^{5-9}$ over medium-sized photoswitches ${ }^{10,11}$ to the dynamics of entire photoactive proteins. ${ }^{12,13}$ They can be used with a variety of excited-state methods, e.g., the complete active space self-consistent field (CASSCF) method, ${ }^{14}$ the algebraicdiagrammatic construction $(\operatorname{ADC}(2)),{ }^{15}$ several coupled cluster methods (e.g., CC2), ${ }^{16}$ as well as time-dependent density functional theory (TDDFT). ${ }^{17,18}$ Also, triplet states ${ }^{19}$ can be included.

However, the greatest challenge remains the large computational cost of NAMD simulations, which is a result of the expensive excited-state methods mentioned above and the fact that $\mathrm{TSH}$ requires not only one but a series of trajectories to determine observables as ensemble averages. This problem can be tackled by using semiempirical methods, ${ }^{5,6}$ employing exciton models ${ }^{20}$ or using graphics processing units (GPUs). The latter have been applied to ground-state properties, ${ }^{21-28}$ excited-state calculations, ${ }^{9,20,29-31}$ ab initio multiple spawning (AIMS), ${ }^{32-35}$ and NAMD $^{36,37}$ simulations.

Based on our recent work, ${ }^{9}$ we explore in our present work the use of simplified TDDFT schemes ${ }^{38,39}$ and the HammesSchiffer-Tully (HST) ${ }^{2}$ model in addition to GPU-based integral routines. They tackle the two major bottlenecks of NAMD: The calculation of the state energies and the nonadiabatic couplings between the states. After a brief summary of the corresponding theory and its validation for the investigated problems, we show timings and use our approach to simulate the photoinduced rotation of the retinal chromophore in the rhodopsin protein at the TDDFT/MM level of theory. Details on the methods (thresholds, convergence criteria, etc.) implemented in our FermiONs++ program package $e^{25,26}$ and the computational setup can be found in the Supporting Information.

In $\mathrm{TSH}_{,}{ }^{1,2}$ a system is allowed to switch the potential energy surface (PES) within one trajectory. The occurrence of such a surface hop depends on the hopping probability: if it exceeds a randomly drawn number between 0 and 1 , the trajectory continues on a different PES with a rescaled nuclear velocity. The average of multiple trajectories with a different series of random numbers describes the behavior of the system. The hopping probability itself is calculated from the change of the state energies and the nonadiabatic couplings $(\mathbf{Q})$, which can also be obtained as the product of the nonadiabatic coupling vectors $(\boldsymbol{\tau})$ and the nuclear velocity $(\dot{\mathbf{R}})$

$$
Q_{I J}=\left\langle\Phi_{I} \mid \frac{\partial}{\partial t} \Phi_{J}\right\rangle=\left\langle\Phi_{I} \mid \frac{\partial}{\partial \mathbf{R}} \Phi_{J}\right\rangle \cdot \dot{\mathbf{R}}=\tau_{I J} \cdot \dot{\mathbf{R}}
$$

Received: January 30, 2020

Accepted: April 23, 2020

Published: May 6, 2020 
where $\Phi_{I}$ is the wave function of the electronic state $I$. While $\tau$ can be calculated using response theory, $\mathbf{Q}$ cannot be determined analytically.

Energies of all considered states, gradients, and $\mathbf{Q}$ are thus the main ingredients of a TSH algorithm. The first can be obtained from TDDFT by solving the TDDFT or random phase approximation (RPA) equations ${ }^{17,40,41}$

$$
\left[\begin{array}{ll}
\mathbf{A} & \mathbf{B} \\
\mathbf{B}^{*} & \mathbf{A}^{*}
\end{array}\right]\left(\begin{array}{l}
\mathbf{X}_{I} \\
\mathbf{Y}_{I}
\end{array}\right)=\omega_{I}\left[\begin{array}{ll}
1 & 0 \\
0 & -1
\end{array}\right]\left(\begin{array}{l}
\mathbf{X}_{I} \\
\mathbf{Y}_{I}
\end{array}\right)
$$

with $\omega_{I}$ being the excitation energy of state $I$. $\mathbf{A}$ and $\mathbf{B}$ are the orbital rotation Hessians, and $\mathbf{X}_{I}$ and $\mathbf{Y}_{I}$ are the transition densities for excitation and de-excitation, respectively. Neglecting $\mathbf{B}$ in eq 2 is known as the Tamm-Dancoff approximation (TDA), ${ }^{42}$ leading to

$$
\mathbf{A X}_{I}=\omega_{I} \mathbf{X}_{I}
$$

The calculation of excitation energies with eqs 2 and 3 is time-consuming, mainly because of the evaluation of the twoelectron integrals in A and B. To accelerate these calculations, we apply the simplified RPA and TDA methods by Grimme and co-workers. ${ }^{38,39,43}$ Here, Coulomb and exchange kernels are approximated ( $\mathbf{J}^{\prime}$ for Coulomb and $\mathbf{K}^{\prime}$ for exchange) using the Mataga-Nishimoto-Ohno-Klopman ${ }^{44-46}$ damped Coulomb operators together with the transition/charge density monopoles $q$ obtained from a Löwdin population analysis ${ }^{47}$

$$
\begin{aligned}
& \mathbf{J}_{p q r s}^{\prime}=\sum_{N M} q_{p q}^{N}\left(\frac{1}{r_{N M}^{\beta}+\left(c_{\mathrm{x}} \eta_{N M}\right)^{-\beta}}\right)^{1 / \beta} q_{r s}^{M} \\
& \mathbf{K}_{p q r s}^{\prime}=\sum_{N M} q_{p q}^{N}\left(\frac{1}{r_{N M}^{\alpha}+\eta_{N M}^{-\alpha}}\right)^{1 / \alpha} q_{r s}^{M}
\end{aligned}
$$

$p, q, \ldots$ are arbitrary molecular orbitals. $r$ is the interatomic distance, $\eta$ is the mean of the chemical hardness of the atoms $N$ and $M . \alpha$ and $\beta$ are global fit parameters, while $c_{\mathrm{x}}$ is the amount of exact exchange. This leads to the following approximate orbital rotation Hessians

$$
\begin{aligned}
& A_{i a j b}^{\prime}=\delta_{i j} \delta_{a b}\left(\epsilon_{a}-\epsilon_{i}\right)+s_{k} K_{i a j b}^{\prime}-J_{i j a b}^{\prime} \\
& B_{i a j b}^{\prime}=s_{k} K_{i a b j}^{\prime}-c_{\mathrm{x}} K_{i b a j}^{\prime}
\end{aligned}
$$

$i, j, \ldots$ denote occupied molecular orbitals, and $a, b, \ldots$ denote virtual molecular orbitals. $s_{k}$ is 2 or 0 for singlet-singlet or singlet-triplet excitations, and $\epsilon_{p}$ is the orbital energy of $p$.

The excitation energies $\left(\omega^{\prime}\right)$ are then obtained by diagonalizing $\mathbf{A}^{\prime}$ in the case of STDA or $\left(\mathbf{A}^{\prime}-\mathbf{B}^{\prime}\right)^{1 / 2}\left(\mathbf{A}^{\prime}+\right.$ $\left.\mathbf{B}^{\prime}\right)\left(\mathbf{A}^{\prime}-\mathbf{B}^{\prime}\right)^{1 / 2}$ in the case of sRPA. To avoid the diagonalization of the entire matrix, the number of included configuration-state functions (CSFs) is truncated using the thresholds $\vartheta_{\mathrm{pCSF}}, \vartheta_{\mathrm{sCSF}}$, and $\vartheta_{\mathrm{CSF}}{ }^{38}$ Only primary (with an energy below $\vartheta_{\text {pCSF }}$ ) and secondary CSFs (with an energy between $\vartheta_{\mathrm{pCSF}}$ and $\vartheta_{\mathrm{CSF}}$ and a significant coupling to the primary CSFs $>\vartheta_{\mathrm{sCSF}}$ ) are considered. The sTDA scheme greatly reduces the cost of excited-state calculations, giving access to absorption spectra of large molecular systems. ${ }^{38}$ The sRPA scheme yields better transition densities, ${ }^{39}$ leading to better transition dipole moments and even enabling the calculation of higher order dynamical response properties. ${ }^{48}$

Excited-state gradients $\left(\boldsymbol{\omega}_{I}^{\mathrm{x}}\right)$ and $\boldsymbol{\tau}$ 's at the TDDFT/TDA level of theory can be derived from eqs 2 and 3 using linear response theory. ${ }^{49-59}$ These equations cannot easily be transferred to sTDA/sRPA, as the derivatives of $\mathbf{J}^{\prime}$ and $\mathbf{K}^{\prime}$ with respect to the nuclear coordinates have, so far, not been implemented. Instead, we are using the calculated $\omega_{I}{ }^{\prime}, \mathbf{X}_{I}^{\prime}$, and $\mathbf{Y}_{I}^{\prime}$ in the RPA/TDA algorithms for $\boldsymbol{\omega}_{I}^{\mathrm{x}}$ and $\boldsymbol{\tau}$ featuring exact evaluations of the two-electron integrals, i.e., without the Mataga-Nishimoto-Ohno-Klopman damped Coulomb operators. As a consequence of this, we expect a slight disagreement between the calculated energies and the excited-state properties ( $\omega_{I}^{\mathrm{x}}$ and $\boldsymbol{\tau}^{\prime}$ s). To validate this approach, we compare optimized structures of biphenyl (I) using the $S_{1}$ potential energy surface at the TDDFT and sTDDFT levels of theory in Table 1 and show $\omega^{\mathrm{x}}$ s and $\boldsymbol{\tau}$ 's of optimized ground-

Table 1. Comparison of Optimized Structures ${ }^{a}$ of Biphenyl (I) Calculated at RPA, TDA, sRPA, and sTDA (PBE0/def2SVP) Levels of Theory Listing the Central C-C Distance (c) and the Dihedral $\gamma$

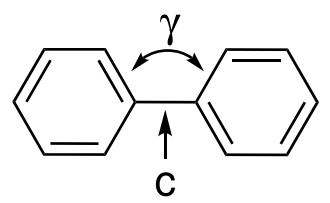

$\begin{array}{ccccc} & \text { RPA } & \text { TDA } & \text { sRPA } & \text { sTDA } \\ c[\AA] & 1.42 & 1.44 & 1.44 & 1.44 \\ |\gamma|[\mathrm{deg}] & 0.02 & 0.03 & 0.02 & 0.01 \\ { }^{a} \text { Using the } S_{1} \text { potential energy surface. } & & \end{array}$

state structures of protonated formaldimine (II) and the Schiff base of the retinal chromophore (III) in Figure 1. A validation based on NAMD is discussed below. Additionally, selected natural transition orbitals ${ }^{60}$ and a screening of the thresholds $\left(\vartheta_{\mathrm{pCSF}}, \vartheta_{\mathrm{CSF}}\right.$, and $\left.\vartheta_{\mathrm{SCSF}}\right)$ are shown in the Supporting Information.

All four optimized $S_{1}$ structures of $\mathbf{I}$ in Table 1 are nearly planar and feature a similar central $\mathrm{C}-\mathrm{C}$ distance. The $\boldsymbol{\omega}^{\mathrm{x}}$ s and $\boldsymbol{\tau}$ 's based on sTDDFT results in Figure 1 are also in good agreement with the RPA and TDA properties. The only differences are the weaker nonadiabatic couplings of II and III and the fact that $\boldsymbol{\omega}_{1}^{\mathrm{x}}$ of III has a larger contribution in the sixmembered ring and a smaller contribution in the conjugated system. Both are the result of the slightly different excitation energies (II: $9.76 \mathrm{eV}$ (RPA), $9.87 \mathrm{eV}$ (sRPA); III: $2.74 \mathrm{eV}$ (RPA), $3.17 \mathrm{eV}$ (sTDA)) and transition densities. In the case of III, three other observations can be made (see Figures S810 in the Supporting Information): (1) sRPA performs worse than sTDA, (2) $\mathrm{PBE0}^{61-64} / \mathrm{def} 2-\mathrm{SVP}^{65,66}$ is, in contrast to $\omega \mathrm{B} 97^{67} /$ def2-SVP, not able to capture the charge transfer character of the excitation, and (3) the natural transition orbitals at the RPA and sTDA levels of theory are nearly identical, indicating that the major source of error is the excitation energy. Table 1 and Figure 1 suggest that simplified TDDFT might also be used for NAMD simulations, which require, however, time-consuming calculations of the Q's between the states. To circumvent the analytical calculation of $\mathbf{Q}$ via $\boldsymbol{\tau}$, we apply the numerical HST model ${ }^{2}$ 
(a) RPA

(b) SRPA
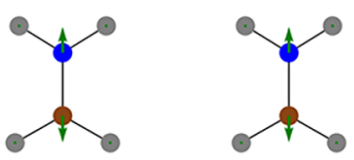

(e) RPA

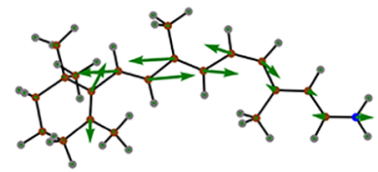

(g) RPA
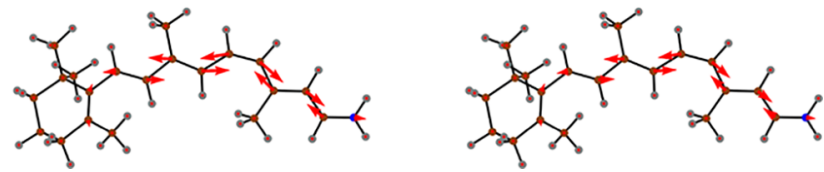

Figure 1. $(a-d) R P A$ and sRPA excited-state gradients of the second excited state $(a+b$, green) and nonadiabatic coupling vectors between the ground and the second excited state $(c+d$, red $)$ of II at the PBE0/def2-SVP level of theory. $(\mathrm{e}-\mathrm{h}) \mathrm{RPA}$ and sTDA excitedstate gradients of the first excited state $(e+f$, green $)$ and nonadiabatic coupling vectors between the ground and the first excited state $(g+h$, red) of III at the $\omega$ B97/def2-SVP level of theory. All calculations have been performed at optimized ground-state geometries.

$$
\begin{aligned}
Q_{I J}\left(t+\frac{\Delta t}{2}\right) & \approx \frac{1}{2 \Delta t}\left[O_{I J}(t, t+\Delta t)-O_{I J}(t+\Delta t, t)\right] \\
& =\frac{1}{2 \Delta t}\left[\left\langle\Phi_{I}(t) \mid \Phi_{J}(t+\Delta t)\right\rangle-\left\langle\Phi_{I}(t+\Delta t) \mid \Phi_{J}(t)\right\rangle\right]
\end{aligned}
$$

Assuming that the excited-state wave functions can be obtained from the ground-state Kohn-Sham orbitals $\left(\phi_{p}\right)$ and the transition densities, $O_{0 I}$ and $O_{I J}$ take the following form

$$
O_{0 I}\left(t_{1}, t_{2}\right)=\sum_{i a} \gamma_{i a}^{0 I}\left(t_{1}\right) S_{i a}\left(t_{1}, t_{2}\right)
$$

$$
\begin{aligned}
O_{I J}\left(t_{1}, t_{2}\right)= & \sum_{p q} \gamma_{p q}^{I J}\left(t_{1}\right) S_{p q}\left(t_{1}, t_{2}\right)+\sum_{i a} X_{i a}^{I}\left(t_{1}\right) X_{i a}^{J}\left(t_{2}\right) \\
& -\sum_{i a} Y_{i a}^{I}\left(t_{1}\right) Y_{i a}^{J}\left(t_{2}\right)
\end{aligned}
$$

with

$$
\begin{aligned}
& S_{p q}\left(t_{1}, t_{2}\right)=\left\langle\phi_{p}\left(t_{1}\right) \mid \phi_{q}\left(t_{2}\right)\right\rangle \\
& \gamma_{0 I}=\mathbf{L}_{I} \\
& \gamma_{I J}=\frac{1}{2}\left(\begin{array}{cc}
-\left(\mathbf{R}_{I}^{T} \mathbf{L}_{J}+\mathbf{L}_{I}^{T} \mathbf{R}_{J}\right)_{i j} & 0 \\
0 & \left(\mathbf{R}_{I} \mathbf{L}_{J}^{T}+\mathbf{L}_{I} \mathbf{R}_{J}^{T}\right)_{a b}
\end{array}\right)
\end{aligned}
$$

$\mathbf{R}_{I}$ and $\mathbf{L}_{I}$ are $\left(\mathbf{X}_{I}+\mathbf{Y}_{I}\right)$ and $\left(\mathbf{X}_{I}-\mathbf{Y}_{I}\right)$, respectively, in the case of RPA and $\mathbf{X}_{I}$ in the case of TDA. The HST model is nowadays widely used in NAMD simulations, ${ }^{68-70}$ because it reduces the computational time (as shown below) and leads to more stable trajectories in the vicinity of conical intersections. $^{71-73}$

A validation of the presented numerical scheme for $\mathbf{Q}$ used in the HST model is shown in the Supporting Information, where we have calculated numerical and analytical $\boldsymbol{\tau}$ 's for formaldehyde. Additionally, we have performed NAMD simulations of II, using the HST model and analytically calculated $\tau$ 's as well as RPA, TDA, sRPA, and sTDA. After excitation to the $S_{2}$ state, the molecule shows a fast conversion to the $S_{1}$ state, which goes along with the elongation of the C$\mathrm{N}$ bond. Further relaxation to the $\mathrm{S}_{0}$ state is achieved via a rotation around this bond. In Figure 2, the increase $\left(S_{2} \rightarrow S_{1}\right)$ and decrease $\left(S_{1} \rightarrow S_{0}\right)$ of the $S_{1}$ occupation is shown. The change of occupation for all states as well as energies and Q's of selected trajectories are listed in the Supporting Information.

In all sets of trajectories, we observe a similar behavior of II, indicating that the HST model and the simplified TDDFT are valid approximations for this example. This is reflected in the $S_{1}$ occupations, which are very similar for all cases. The only differences are slightly different $S_{2}-S_{1}$ nonadiabatic couplings when applying the HST model and a slower decay of the $S_{1}$ occupation in the case of the simplified TDDFT methods, which is, however, also visible in the case of TDA. The first observation is in good agreement with previous findings ${ }^{71-73}$ comparing analytical and numerical Q's in the vicinity of (a)

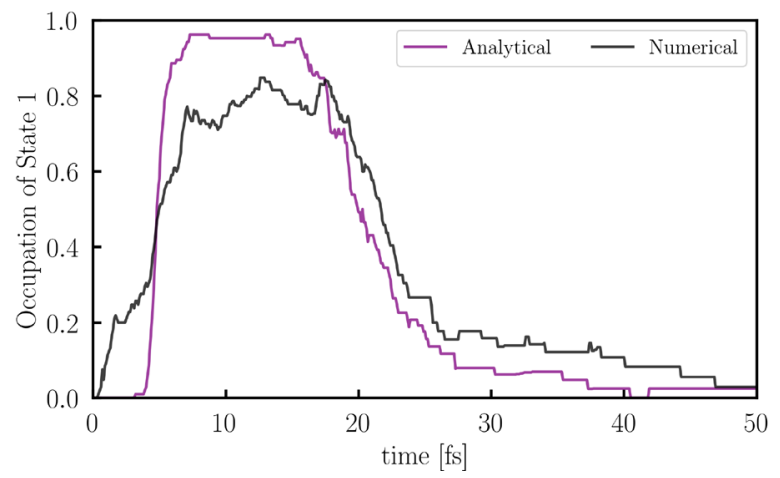

(b)

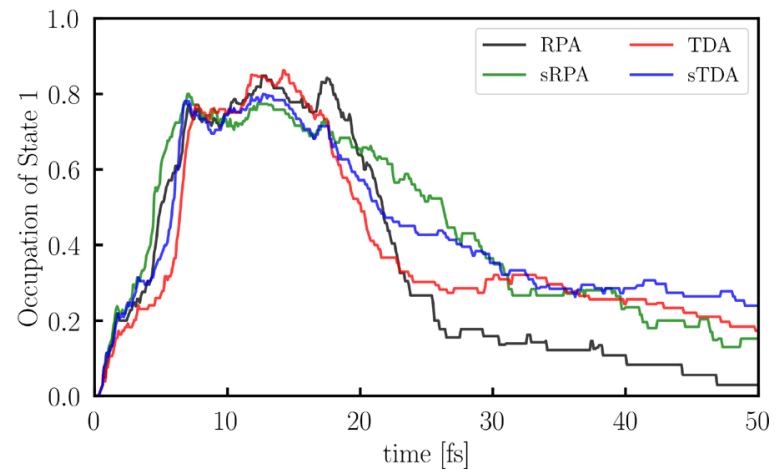

Figure 2. Change of $S_{1}$ state occupations of protonated formaldimine (II) calculated as an average of all NAMD simulations at (a) the RPA (PBE0/def2-SVP) level of theory using analytical and numerical nonadiabatic couplings and (b) RPA, TDA, sRPA, and sTDA (PBE0/def2-SVP) results using numerical nonadiabatic couplings. 
Table 2. Computation Times of Ground-State Energy $\left(E_{0}\right)$ and Gradient $\left(E_{0}^{\mathrm{x}}\right)$, Excited-State Energies $\left(\omega_{I}\right)$ and Gradient $\left(\omega_{1}^{\mathrm{x}}\right)$, and Nonadiabatic Couplings (Q) Calculations of the Schiff Base of the Retinal Chromophore (III) at the RPA and sRPA (PBE0/def2-SVP) Levels of Theory, Using a Different Number of Roots $\left(N_{\text {roots }}\right)$ and Nonadiabatic Couplings $\left(\frac{\left(N_{\text {roots }}+1\right) N_{\text {roots }}}{2}\right)$ as Well as Analytical and Numerical Q's ${ }^{a}$

\begin{tabular}{|c|c|c|c|c|c|c|c|}
\hline$N_{\text {roots }}$ & Q & RPA/sRPA & $t\left(E_{0}\right)+t\left(E_{0}^{\mathrm{x}}\right)$ & $t\left(\omega_{I}\right)$ & $t\left(\omega_{1}^{x}\right)$ & $t(\mathbf{Q})$ & $t($ total $)$ \\
\hline 2 & analytical* & RPA* & $34 \mathrm{~s}$ & $28 \mathrm{~s}$ & $101 \mathrm{~s}$ & $201 \mathrm{~s}$ & $\sim 6 \min$ \\
\hline 2 & analytical & RPA & $26 \mathrm{~s}$ & $19 \mathrm{~s}$ & $62 \mathrm{~s}$ & $128 \mathrm{~s}$ & $\sim 4 \mathrm{~min}$ \\
\hline 2 & numerical & RPA & $26 \mathrm{~s}$ & $19 \mathrm{~s}$ & $62 \mathrm{~s}$ & $<1 \mathrm{~s}$ & $\sim 2 \min$ \\
\hline 2 & numerical & sRPA & $26 \mathrm{~s}$ & $<1 \mathrm{~s}$ & $62 \mathrm{~s}$ & $<1 \mathrm{~s}$ & $\sim 1.5 \mathrm{~min}$ \\
\hline 3 & analytical & RPA & $26 \mathrm{~s}$ & $36 \mathrm{~s}$ & $62 \mathrm{~s}$ & $309 \mathrm{~s}$ & $\sim 7 \mathrm{~min}$ \\
\hline 3 & numerical & $\mathrm{RPA}$ & $26 \mathrm{~s}$ & $36 \mathrm{~s}$ & $62 \mathrm{~s}$ & $<1 \mathrm{~s}$ & $\sim 2 \min$ \\
\hline 3 & numerical & sRPA & $26 \mathrm{~s}$ & $<1 \mathrm{~s}$ & $62 \mathrm{~s}$ & $<1 \mathrm{~s}$ & $\sim 1.5 \mathrm{~min}$ \\
\hline 7 & analytical & RPA & $26 \mathrm{~s}$ & $51 \mathrm{~s}$ & $62 \mathrm{~s}$ & $1822 \mathrm{~s}$ & $\sim 32.5 \mathrm{~min}$ \\
\hline 7 & numerical & RPA & $26 \mathrm{~s}$ & $51 \mathrm{~s}$ & $62 \mathrm{~s}$ & $<1 \mathrm{~s}$ & $\sim 2.5 \mathrm{~min}$ \\
\hline 7 & numerical & sRPA & $26 \mathrm{~s}$ & $<1 \mathrm{~s}$ & $62 \mathrm{~s}$ & $<1 \mathrm{~s}$ & $\sim 1.5 \mathrm{~min}$ \\
\hline
\end{tabular}

${ }^{a}$ Asterisks mark calculations that have been performed entirely on CPUs. All calculations were conducted on two Intel Xeon CPU E5 2640 v4@ $2.20 \mathrm{GHz}$ (20 threads) CPUs and four AMD FirePro 3D W8100 GPUs.

conical intersections. The latter trend is also reflected in the Q's and $\boldsymbol{\tau}$ 's.

Here, we want to stress that one of the well-known limitations of TDDFT is its poor description of conical intersections involving the ground state, ${ }^{74,75}$ which can be improved by applying, e.g., the TDA. ${ }^{76,77}$ Alternatively, the spin-restricted ensemble-referenced KS (REKS) $\operatorname{method}^{78}$ can be employed. Obviously, these limitations are not overcome by applying the simplified TDDFT approach. However, for this particular system, good agreement between TDDFT and CASSCF simulations has been observed, ${ }^{70}$ so that we are confident that our chosen setup allows for a good qualitative description of the investigated processes. Please note that the simulations using sTDA or sRPA are not strictly energy conserving as discussed above. Therefore, the standard deviation of the total energy is approximately 5 times higher than in standard TDDFT simulations. However, rescaling the nuclear velocities to enforce energy conservation has no effect on the average properties of the system, which illustrates the validity of the present approach.

Table 2 presents the impact of the approximations on the performance of an NAMD simulation of III. It shows that HST and sRPA virtually eliminate the computational cost of the calculations of $\omega_{I}^{\mathrm{x}} \mathrm{s}$ and $\mathbf{Q}$ 's. In combination with the GPUbased integral evaluations, the total speed-up in the case of $N_{\text {roots }}=2$ is $\sim 4$ with respect to the CPU-based RPA implementation using analytical $\boldsymbol{\tau}$ 's. An NAMD simulation of III involving 5000 steps (e.g., 1 ps simulation using 0.2 fs time steps) can thus be conducted within $\sim 5$ instead of $\sim 21$ days. The acceleration becomes even larger when more excited states and nonadiabatic couplings are considered (e.g., a factor of more than 20 for III in case of $N_{\text {roots }}=7$ ). This and the fact that the performance of GPUs is better for large molecular systems (see, ref 9) makes the presented approach interesting for the investigation of systems involving hundreds of atoms and plenty of electronic states.

As a first application of our proposed scheme, we have calculated 105 NAMD simulations of the rhodopsin protein (IV) at the sTDA/MM $(\omega \mathrm{B} 97 /$ def2-SVP $)$ level of theory. The chromophore of IV (Figure 3a) undergoes a cis-trans isomerization when exposed to light (see Figure 3c). For a (a)

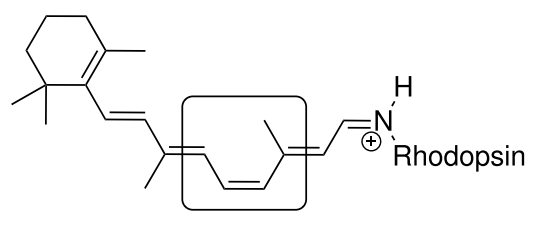

(b)<smiles>C=Cc1cccc(=CC)c1=CC</smiles>

(c)
Figure 3. (a) Structure of the chromophore of rhodopsin (IV). (b) Important dihedrals $\left(\gamma_{1}\right.$ and $\left.\gamma_{2}\right)$ in and (c) the bicycle pedal isomerization mechanism of IV. The part of the molecule shown in (b) and (c) is located by the rectangle in (a).

review of calculations on this system, the reader is referred to ref 79. Similar systems have also been investigated by Martínez et al. ${ }^{34,35}$ The change of the dihedral $\gamma_{1}$ (defined in Figure $3 \mathrm{~b}$ ) and the state occupations are shown in Figure 4.

Out of 105 calculated trajectories, 9 feature a cis-trans isomerization (see Figure 4. A movie of the isomerization is available at https://www.cup.uni-muenchen.de/pc/ ochsenfeld/download/). Most of them reach $\gamma_{1}=-90^{\circ}$ at $\sim 280 \mathrm{fs}$, which coincides with the crossing point of the state occupations (see Figure 4). This hop time $(t)$ is significantly higher, and the yield $(y)$ of $9 \%$ significantly lower than the experimental $(t=147.7 \pm 1.0$ fs; $y=0.63 \pm 0.01)$ results reported in ref 13 . Our analysis of III (see Figure 1) indicates that the weaker gradients and nonadiabatic couplings of sTDA or the discussed problems of TDDFT with conical intersections (see also refs 79-81) may be the reason for this. However, our approach describes the direction and mechanism (see Figure 3c) of the isomerization correctly. In contrast to previous work, ${ }^{12,13,79}$ our method requires, besides $\alpha, \beta$, and the $\mathrm{QM} / \mathrm{MM}$ ansatz, no further parametrizations and/or reductions of the system. One trajectory of IV takes 5-7 days on two Intel Xeon CPU E5 2640 v4@ $2.20 \mathrm{GHz}$ (20 threads) CPUs and four AMD FirePro 3D W8100 GPUs using our FermiONs++ program package. ${ }^{25,26}$

We have introduced a combination of GPU-based integral routines, simplified TDDFT schemes, and numerical nonadiabatic couplings for efficient NAMD simulations. For all investigated systems ranging from small organic molecules (II) 
(a)

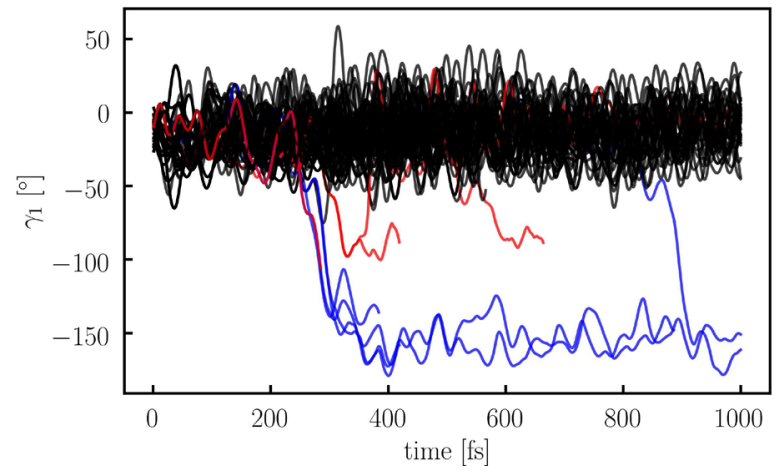

(b)

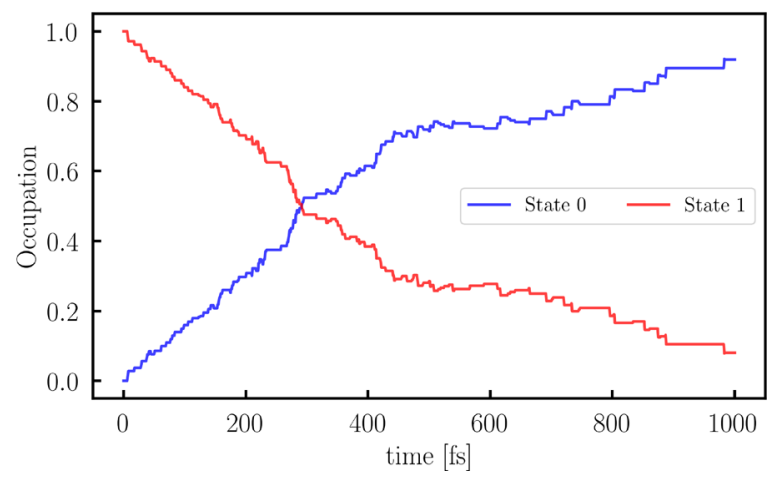

Figure 4. NAMD simulations of IV at the sTDA (PBE0/def2-SVP) level of theory using the HST model: (a) Change of the dihedral $\gamma_{1}$ indicating trajectories with no rotation (black), half rotation (red), and full rotation (blue). (b) Change of the state occupations of IV calculated as an average of all trajectories.

to proteins (IV), excited-state properties and dynamics are described qualitatively correctly with a significantly reduced computational cost. The latter is due to the vanishing computational times for TDDFT energies and nonadiabatic couplings calculations. The present approach may be used to qualitatively explore relaxation pathways and predict trends (e.g., effects of mutations or different isotopes) within these reactions.

\section{ASSOCIATED CONTENT}

\section{(s) Supporting Information}

The Supporting Information is available free of charge at https://pubs.acs.org/doi/10.1021/acs.jpclett.0c00320.

Computational details, a validation of the finite-difference nonadiabatic couplings, a benchmark of sTDA/ sRPA, and the used thresholds for the investigated systems (PDF)

\section{AUTHOR INFORMATION}

\section{Corresponding Author}

Christian Ochsenfeld - Chair of Theoretical Chemistry, Department of Chemistry, University of Munich (LMU), D81377 München, Germany; Max Planck Institute for Solid State Research, D-70569 Stuttgart, Germany; 이이.org/ 0000-0002-4189-6558; Email: c.ochsenfeld@fkf.mpg.de

\section{Authors}

Laurens D. M. Peters - Chair of Theoretical Chemistry, Department of Chemistry, University of Munich (LMU), D81377 München, Germany

Jörg Kussmann - Chair of Theoretical Chemistry, Department of Chemistry, University of Munich (LMU), D-81377 München, Germany; 이이. orcid.org/0000-0002-4724-8551

Complete contact information is available at:

https://pubs.acs.org/10.1021/acs.jpclett.0c00320

\section{Notes}

The authors declare no competing financial interest.

\section{ACKNOWLEDGMENTS}

Financial support was provided by the Innovative Training Network "Computational Spectroscopy In Natural Sciences And Engineering" (ITN-COSINE) and the Deutsche Forschungsgemeinschaft (DFG, German Research Foundation) under Germany's Excellence Strategy - EXC 2089/1390776260. C.O. acknowledges further support as a MaxPlanck-Fellow at the MPI-FKF Stuttgart.

\section{REFERENCES}

(1) Tully, J. C. Molecular Dynamics with Electronic Transitions. J. Chem. Phys. 1990, 93, 1061-1071.

(2) Hammes-Schiffer, S.; Tully, J. C. Proton Transfer in Solution: Molecular Dynamics with Quantum Transitions. J. Chem. Phys. 1994, 101, 4657-4667.

(3) Wang, L.; Akimov, A.; Prezhdo, O. V. Recent Progress in Surface Hopping: 2011-2015. J. Phys. Chem. Lett. 2016, 7, 2100-2112.

(4) Crespo-Otero, R.; Barbatti, M. Recent Advances and Perspectives on Nonadiabatic Mixed Quantum-Classical Dynamics. Chem. Rev. 2018, 118, 7026-7068.

(5) Kazaryan, A.; Lan, Z.; Schäfer, L. V.; Thiel, W.; Filatov, M. Surface Hopping Excited-State Dynamics Study of the Photoisomerization of a Light-Driven Fluorene Molecular Rotary Motor. J. Chem. Theory Comput. 2011, 7, 2189-2199.

(6) Nikiforov, A.; Gamez, J. A.; Thiel, W.; Filatov, M. Computational Design of a Family of Light-Driven Rotary Molecular Motors with Improved Quantum Efficiency. J. Phys. Chem. Lett. 2016, 7, 105110.

(7) Oruganti, B.; Wang, J.; Durbeej, B. Quantum Chemical Design of Rotary Molecular Motors. Int. J. Quantum Chem. 2018, 118, No. e25405.

(8) Wang, J.; Oruganti, B.; Durbeej, B. Light-Driven Rotary Molecular Motors without Point Chirality: A Minimal Design. Phys. Chem. Chem. Phys. 2017, 19, 6952-6956.

(9) Peters, L. D. M.; Kussmann, J.; Ochsenfeld, C. Nonadiabatic Molecular Dynamics on Graphics Processing Units: Performance and Application to Rotary Molecular Motors. J. Chem. Theory Comput. 2019, 15, 6647-6659.

(10) Pederzoli, M.; Pittner, J.; Barbatti, M.; Lischka, H. Nonadiabatic Molecular Dynamics Study of the cis-trans Photoisomerization of Azobenzene Excited to the $S_{1}$ State. J. Phys. Chem. A 2011, $115,11136-11143$.

(11) Jankowska, J.; Barbatti, M.; Sadlej, J.; Sobolewski, A. L. Tailoring the Schiff Base Photoswitching - a Non-Adiabatic Molecular Dynamics Study of Substituent Effect on Excited State Proton Transfer. Phys. Chem. Chem. Phys. 2017, 19, 5318-5325.

(12) Manathunga, M.; Yang, X.; Luk, H. L.; Gozem, S.; Frutos, L. M.; Valentini, A.; Ferrè, N.; Olivucci, M. Probing the Photodynamics of Rhodopsins with Reduced Retinal Chromophores. J. Chem. Theory Comput. 2016, 12, 839-850.

(13) Schnedermann, C.; Yang, X.; Liebel, M.; Spillane, K. M.; Lugtenburg, J.; Fernández, I.; Valentini, A.; Schapiro, I.; Olivucci, M.; Kukura, P.; et al. Evidence for a Vibrational Phase-Dependent Isotope 
Effect on the Photochemistry of Vision. Nat. Chem. 2018, 10, 449455.

(14) Roos, B. A. New Method for Large-Scale Cl Calculations. Chem. Phys. Lett. 1972, 15, 153-159.

(15) Trofimov, A. B.; Krivdina, I. L.; Weller, J.; Schirmer, J. Algebraic-Diagrammatic Construction Propagator Approach to Molecular Response Properties. Chem. Phys. 2006, 329, 1-10.

(16) Christiansen, O.; Koch, H.; Jorgensen, P. The Second-Order Approximate Coupled Cluster Singles and Doubles Model CC2. Chem. Phys. Lett. 1995, 243, 409-418.

(17) Casida, M. E. Time-Dependent Density Functional Response Theory for Molecules. Recent Advances in Density Functional Methods 1995, 1, 155-192.

(18) Casida, M. E. Time-Dependent Density-Functional Theory for Molecules and Molecular Solids. J. Mol. Struct.: THEOCHEM 2009, 914, 3-18.

(19) Mai, S.; Marquetand, P.; González, L. Nonadiabatic Dynamics: The SHARC Approach. WIREs Comput. Mol. Sci. 2018, 8, No. e1370. (20) Sisto, A.; Glowacki, D. R.; Martínez, T. J. Ab Initio Nonadiabatic Dynamics of Multichromophore Complexes: A Scalable Graphical-Processing-Unit-Accelerated Exciton Framework. Acc. Chem. Res. 2014, 47, 2857-2866.

(21) Yasuda, K. Two-Electron Integral Evaluation on the Graphics Processor Unit. J. Comput. Chem. 2008, 29, 334-342.

(22) Ufimtsev, I. S.; Martínez, T. J. Quantum Chemistry on Graphical Processing Units. 1. Strategies for Two-Electron Integral Evaluation. J. Chem. Theory Comput. 2008, 4, 222-231.

(23) Ufimtsev, I. S.; Martínez, T. J. Quantum Chemistry on Graphical Processing Units. 2. Direct Self-Consistent-Field Implementation. J. Chem. Theory Comput. 2009, 5, 1004-1015.

(24) Ufimtsev, I. S.; Martínez, T. J. Quantum Chemistry on Graphical Processing Units. 3. Analytical Energy Gradients, Geometry Optimization, and First Principles Molecular Dynamics. J. Chem. Theory Comput. 2009, 5, 2619-2628.

(25) Kussmann, J.; Ochsenfeld, C. Pre-Selective Screening for Matrix Elements in Linear-Scaling Exact Exchange Calculations. J. Chem. Phys. 2013, 138, 134114.

(26) Kussmann, J.; Ochsenfeld, C. Preselective Screening for LinearScaling Exact Exchange-Gradient Calculations for Graphics Processing Units and General Strong-Scaling Massively Parallel Calculations. J. Chem. Theory Comput. 2015, 11, 918-922.

(27) Kussmann, J.; Ochsenfeld, C. Hybrid CPU/GPU Integral Engine for Strong-Scaling Ab Initio Methods. J. Chem. Theory Comput. 2017, 13, 3153-3159.

(28) Peters, L. D. M.; Kussmann, J.; Ochsenfeld, C. Efficient and Accurate Born-Oppenheimer Molecular Dynamics for Large Molecular Systems. J. Chem. Theory Comput. 2017, 13, 5479-5485.

(29) Isborn, C. M.; Luehr, N.; Ufimtsev, I. S.; Martínez, T. J. Excited-State Electronic Structure with Configuration Interaction Singles and Tamm-Dancoff Time-Dependent Density Functional Theory on Graphical Processing Units. J. Chem. Theory Comput. 2011, 7, 1814-1823.

(30) Hohenstein, E. G.; Luehr, N.; Ufimtsev, I. S.; Martínez, T. J. An Atomic Orbital-Based Formulation of the Complete Active Space Self-Consistent Field Method on Graphical Processing Units. J. Chem. Phys. 2015, 142, 224103.

(31) Snyder, J. W.; Hohenstein, E. G.; Luehr, N.; Martínez, T. J. An Atomic Orbital-Based Formulation of Analytical Gradients and Nonadiabatic Coupling Vector Elements for the State-Averaged Complete Active Space Self-Consistent Field Method on Graphical Processing Units. J. Chem. Phys. 2015, 143, 154107.

(32) Curchod, B. F. E.; Sisto, A.; Martínez, T. J. Ab Initio Multiple Spawning Photochemical Dynamics of DMABN Using GPUs. J. Phys. Chem. A 2017, 121, 265-276.

(33) Snyder, J. W.; Curchod, B. F. E.; Martínez, T. J. GPUAccelerated State-Averaged Complete Active Space Self-Consistent Field Interfaced with $\mathrm{Ab}$ Initio Multiple Spawning Unravels the Photodynamics of Provitamin $\mathrm{D}_{3}$. J. Phys. Chem. Lett. 2016, 7, 24442449.
(34) Liang, R.; Liu, F.; Martínez, T. J. Nonadiabatic Photodynamics of Retinal Protonated Schiff Base in Channelrhodopsin 2. J. Phys. Chem. Lett. 2019, 10, 2862-2868.

(35) Yu, J. K.; Liang, R.; Liu, F.; Martínez, T. J. First-Principles Characterization of the Elusive I Fluorescent State and the Structural Evolution of Retinal Protonated Schiff Base in Bacteriorhodopsin. J. Am. Chem. Soc. 2019, 141, 18193-18203.

(36) Hollas, D.; Šštík, L.; Hohenstein, E. G.; Martínez, T. J.; Slavícek, P. Nonadiabatic Ab Initio Molecular Dynamics with the Floating Occupation Molecular Orbital-Complete Active Space Configuration Interaction Method. J. Chem. Theory Comput. 2018, $14,339-350$.

(37) Filatov, M.; Min, S. K.; Kim, K. W. Direct Nonadiabatic Dynamics by Mixed Quantum-Classical Formalism Connected with Ensemble Density Functional Theory Method: Application to transPenta-2,4-dieniminium Cation. J. Chem. Theory Comput. 2018, 14, 4499-4512.

(38) Grimme, S. A Simplified Tamm-Dancoff Density Functional Approach for the Electronic Excitation Spectra of Very Large Molecules. J. Chem. Phys. 2013, 138, 244104.

(39) Bannwarth, C.; Grimme, S. A Simplified Time-Dependent Density Functional Theory Approach for Electronic Ultraviolet and Circular Dichroism Spectra of Very Large Molecules. Comput. Theor. Chem. 2014, 1040-1041, 45-53.

(40) Bauernschmitt, R.; Ahlrichs, R. Treatment of Electronic Excitations within the Adiabatic Approximation of Time Dependent Density Functional Theory. Chem. Phys. Lett. 1996, 256, 454-464.

(41) Bauernschmitt, R.; Häser, M.; Treutler, O.; Ahlrichs, R. Calculation of Excitation Energies within Time-Dependent Density Functional Theory Using Auxiliary Basis Set Expansions. Chem. Phys. Lett. 1997, 264, 573-578.

(42) Maurice, D.; Head-Gordon, M. Configuration Interaction with Single Substitutions for Excited States of Open-Shell Molecules. Int. J. Quantum Chem. 1995, 56, 361-370.

(43) Risthaus, T.; Hansen, A.; Grimme, S. Excited States Using the Simplified TammDancoff-Approach for Range-Separated Hybrid Density Functionals: Development and Application. Phys. Chem. Chem. Phys. 2014, 16, 14408-14419.

(44) Nishimoto, K.; Mataga, N. Electronic Structure and Spectra of Nitrogen Heterocycles. Z. Phys. Chem. 1957, 13, 140-157.

(45) Ohno, K. Some Remarks on the Pariser-Parr-Pople Method. Theor. Chim. Acta 1964, 2, 219-227.

(46) Klopman, G. A Semiempirical Treatment of Molecular Structures. II. Molecular Terms and Application to Diatomic Molecules. J. Am. Chem. Soc. 1964, 86, 4550-4557.

(47) Löwdin, P.-O. The Nonorthogonality Problem Connected with the Use of Atomic Wave Functions in the Theory of Molecules and Crystals. J. Chem. Phys. 1950, 18, 365-375.

(48) de Wergifosse, M.; Grimme, S. Nonlinear-Response Properties in a Simplified Time-Dependent Density Functional Theory (sTDDFT) Framework: Evaluation of the First Hyperpolarizability. J. Chem. Phys. 2018, 149, 024108.

(49) Furche, F.; Ahlrichs, R. Adiabatic Time-Dependent Density Functional Methods for Excited State Properties. J. Chem. Phys. 2002, $117,7433-7447$.

(50) Send, R.; Furche, F. First-Order Nonadiabatic Couplings from Time-Dependent Hybrid Density Functional Response Theory: Consistent Formalism, Implementation, and Performance. J. Chem. Phys. 2010, 132, 044107.

(51) Fatehi, S.; Alguire, E.; Shao, Y.; Subotnik, J. E. Analytic Derivative Couplings between Configuration-Interaction-Singles States with Built-In Electron-Translation Factors for Translational Invariance. J. Chem. Phys. 2011, 135, 234105.

(52) Ou, Q.; Fatehi, S.; Alguire, E.; Shao, Y.; Subotnik, J. E. Derivative Couplings between TDDFT Excited States Obtained by Direct Differentiation in the Tamm-Dancoff Approximation. J. Chem. Phys. 2014, 141, 024114.

(53) Li, Z.; Liu, W. First-order Nonadiabatic Coupling Matrix Elements between Excited States: A Lagrangian Formulation at the 
CIS, RPA, TD-HF, and TD-DFT Levels. J. Chem. Phys. 2014, 141, 014110.

(54) Li, Z.; Suo, B.; Liu, W. First Order Nonadiabatic Coupling Matrix Elements between Excited States: Implementation and Application at the TD-DFT and pp-TDA Levels. J. Chem. Phys. 2014, 141, 244105.

(55) Ou, Q.; Bellchambers, G. D.; Furche, F.; Subotnik, J. E. FirstOrder Derivative Couplings between Excited States from Adiabatic TDDFT Response Theory. J. Chem. Phys. 2015, 142, 064114.

(56) Ou, Q.; Alguire, E. C.; Subotnik, J. E. Derivative Couplings between Time-Dependent Density Functional Theory Excited States in the Random-Phase Approximation Based on Pseudo-Wavefunctions: Behavior Around Conical Intersections. J. Phys. Chem. B 2015, 119, 7150-7161.

(57) Zhang, X.; Herbert, J. M. Analytic Derivative Couplings in Time-Dependent Density Functional Theory: Quadratic Response Theory Versus Pseudo-Wavefunction Approach. J. Chem. Phys. 2015, 142, 064109.

(58) Parker, S. M.; Roy, S.; Furche, F. Unphysical Divergences in Response Theory. J. Chem. Phys. 2016, 145, 134105.

(59) Zhang, X.; Herbert, J. M. Analytic Derivative Couplings for Spin-Flip Configuration Interaction Singles and Spin-Flip TimeDependent Density Functional Theory. J. Chem. Phys. 2014, 141, 064104.

(60) Martin, R. L. Natural Transition Orbitals. J. Chem. Phys. 2003, 118, 4775-4777.

(61) Perdew, J. P.; Burke, K.; Ernzerhof, M. Generalized Gradient Approximation Made Simple. Phys. Rev. Lett. 1996, 77, 3865-3868.

(62) Perdew, J. P.; Burke, K.; Ernzerhof, M. Generalized Gradient Approximation Made Simple [Erratum]. Phys. Rev. Lett. 1997, 78, No. 1396(E).

(63) Ernzerhof, M.; Scuseria, G. E. Assessment of the Perdew-BurkeErnzerhof Exchange-Correlation Functional. J. Chem. Phys. 1999, 110, 5029-5036.

(64) Adamo, C.; Barone, V. Toward Reliable Density Functional Methods without Adjustable Parameters: The PBE0Model. J. Chem. Phys. 1999, 110, 6158-6170.

(65) Weigend, F.; Ahlrichs, R. Balanced Basis Sets of Split Valence, Triple Zeta Valence and Quadruple Zeta Valence Quality for H to Rn: Design and Assessment of Accuracy. Phys. Chem. Chem. Phys. 2005, 7, 3297-3305.

(66) Weigend, F. Accurate Coulomb-Fitting Basis Sets for H to Rn. Phys. Chem. Chem. Phys. 2006, 8, 1057-1065.

(67) Chai, J. D.; Head-Gordon, M. Systematic Optimization of Long-Range Corrected Hybrid Density Functionals. J. Chem. Phys. 2008, 128, 084106.

(68) Tapavicza, E.; Tavernelli, I.; Rothlisberger, U. Trajectory Surface Hopping within Linear Response Time-Dependent DensityFunctional Theory. Phys. Rev. Lett. 2007, 98, 023001.

(69) Pittner, J.; Lischka, H.; Barbatti, M. Optimization of Mixed Quantum-Classical Dynamics: Time-derivative Coupling Terms and Selected Couplings. Chem. Phys. 2009, 356, 147-152.

(70) Tavernelli, I.; Tapavicza, E.; Rothlisberger, U. Non-Adiabatic Dynamics Using Time-Dependent Density Functional Theory: Assessing the Coupling Strengths. J. Mol. Struct.: THEOCHEM 2009, 914, 22-29.

(71) Plasser, F.; Crespo-Otero, R.; Pederzoli, M.; Pittner, J.; Lischka, H.; Barbatti, M. Surface Hopping Dynamics with Correlated SingleReference Methods: 9H-Adenine as a Case Study. J. Chem. Theory Comput. 2014, 10, 1395-1405.

(72) Meek, G. A.; Levine, B. G. Evaluation of the Time-Derivative Coupling for Accurate Electronic State Transition Probabilities from Numerical Simulations. J. Phys. Chem. Lett. 2014, 5, 2351-2356.

(73) Jain, A.; Alguire, E.; Subotnik, J. E. An Efficient, Augmented Surface Hopping Algorithm That Includes Decoherence for Use in Large-Scale Simulations. J. Chem. Theory Comput. 2016, 12, 52565268.

(74) Casida, M. E.; Gutierrez, F.; Guan, J.; Gadea, F.-X.; Salahub, D.; Daudey, J.-P. Charge-Transfer Correction for Improved Time-
Dependent Local Density Approximation Excited-State Potential Energy Curves: Analysis within the Two-Level Model with Illustration for $\mathrm{H} 2$ and LiH. J. Chem. Phys. 2000, 113, 7062-7071.

(75) Levine, B. G.; Ko, C.; Quenneville, J.; Martínez, T. J. Conical Intersections and Double Excitations in Time-Dependent Density Functional Theory. Mol. Phys. 2006, 104, 1039-1051.

(76) Cordova, F.; Doriol, L. J.; Ipatov, A.; Casida, M. E.; Filippi, C.; Vela, A. Troubleshooting Time-Dependent Density-Functional Theory for Photochemical Applications: Oxirane. J. Chem. Phys. 2007, 127, 164111.

(77) Tapavicza, E.; Bellchambers, G. D.; Vincent, J. C.; Furche, F. $\mathrm{Ab}$ Initio Non-Adiabatic Molecular Dynamics. Phys. Chem. Chem. Phys. 2013, 15, 18336-18348.

(78) Filatov, M.; Shaik, S. A Spin-Restricted Ensemble-Referenced Kohn-Sham Method and Its Application to Diradicaloid Situations. Chem. Phys. Lett. 1999, 304, 429-437.

(79) Gozem, S.; Luk, H. L.; Schapiro, I.; Olivucci, M. Theory and Simulation of the Ultrafast Double-Bond Isomerization of Biological Chromophores. Chem. Rev. 2017, 117, 13502-13565.

(80) Huix-Rotllant, M.; Filatov, M.; Gozem, S.; Schapiro, I.; Olivucci, M.; Ferré, N. Assessment of Density Functional Theory for Describing the Correlation Effects on the Ground and Excited State Potential Energy Surfaces of a Retinal Chromophore Model. J. Chem. Theory Comput. 2013, 9, 3917-3932.

(81) Valsson, O.; Filippi, C.; Casida, M. E. Regarding the Use and Misuse of Retinal Protonated Schiff Base Photochemistry as a Test Case for Time-Dependent Density-Functional Theory. J. Chem. Phys. 2015, 142, 144104. 